\title{
The influence of antibiotics and dietary components on gut microbiota
}

\author{
Ruth K. Dudek-Wicher, Adam Junka, Marzenna Bartoszewicz \\ Department of Pharmaceutical Microbiology and Parasitology, Medical University of Wroclaw, Wroclaw, Poland
}

Gastroenterology Rev 2018; 13 (2): 85-92

DOI: https://doi.org/10.5114/pg.2018.76005

Key words: microbiota, antibiotics, diet.

Address for correspondence: Ruth K. Dudek-Wicher, Department of Pharmaceutical Microbiology and Parasitology, Medical University of Wroclaw, 211 Borowska St, 50-556 Wroclaw, Poland, phone: +48 606763 589, e-mail: r.dudek.wicher@gmail.com

\begin{abstract}
The gut microbiota acts as a real organ. It exerts important metabolic functions, and regulates the inflammatory response by stimulating the immune system. Gut microbial imbalance (dysbiosis) has been linked to important human diseases and inflammation-related disorders. The symbiotic interactions between resident microorganisms and the gastrointestinal tract significantly contribute to maintaining gut homeostasis. The present review summarizes our knowledge regarding the impact of different antibiotics causing such long-term consequences as decreased microbial diversity, modulation of the Bacteroidetes/Firmicutes ratio, Clostridium difficile overgrowth, and increased expansion of the opportunistic pathogens Salmonella typhimurium, Escherichia spp., and Klebsiella spp. Also, food additives, such as emulsifiers and artificial sweeteners, which are meant to reduce the risk of obesity and diabetes, may actually increase the risk of diseases due to microbial alterations. On the other hand, dietary components such as polyphenols, omega-3 acids or curcumin may positively affect gut microbiota composition.
\end{abstract}

\section{Introduction}

The human microbiome contains ten times as many cells as the rest of the human body. In recent years the gut microbiota has been a subject of increased interest within the scientific community. Bacterial colonization of the intestine is critical to the normal development of many functional aspects. Abnormalities in the gut microbiota have been associated with many common diseases such as allergies, metabolic diseases including obesity and diabetes, cardiovascular diseases [1], inflammatory bowel disease (IBD) [2], irritable bowel syndrome (IBS) [3], neurodegenerative disorders [4], cystic fibrosis [5] and kidney stones [6]. The majority of the gut bacteria are non-pathogenic and coexist with the enterocytes in a symbiotic relationship. Many studies have shown that the gut microbiota has a fundamental role in maintaining homeostasis not only in the intestines but throughout the entire human organism. High-quality data from the US Human Microbiome Project (HMP) have demonstrated the beneficial functions of the normal gut flora on health down to the genetic level. For example, studies have identified several microbial genes which allow intestinal bacteria to enter into an intense digestive process that takes place in the human digestive tract. Chromosomally encoded HMO-related gene cluster 1 , responsible for human milk oligosaccharide digestion, is one of the best investigated proofs of the aforementioned phenomenon [7].

The scope of this manuscript is to review selected studies from the period 2011-2017, investigating the alterations in the gut microbiota caused by antibiotics and diet ingredients and the impact on human health. Search criteria were based on 33 research papers, found in such medical databases as PubMed, Scopus and Science Direct.

\section{The role of healthy gut microbiota}

Gut microbiota refers to the entire population of microorganisms that colonizes the intestines. It includes an enormous variety of bacteria, fungi, archaea, viruses, and protozoans. A healthy gut microbiota is predominantly constituted by the phyla Firmicutes and Bacteroidetes followed by Actinobacteria and Verrucomicrobia. These phyla remain constant, but in each part of the intestines, extensive diversity, both qualitative and quantitative, of the genera is observed. 
The species occurring in the gut microbiota are: Gemella spp., Megasphaera spp., Pseudomonas spp., Prevotella spp., Streptococcus spp., Rothia spp., Veillonella spp., Clostridium spp., Porphyromonas spp., Eubacterium spp., Ruminococcus spp., Enterobacter spp., Enterococcus spp., Lactobacillus spp., Peptostreptococcus spp., Fusobacteria spp., Lachnospira spp., Roseburia spp., Butyrivibrio spp., Faecalibacterium spp., Proteobacteria spp. [8].

Normally, these microbes are commensal or mutualists and predominantly aid in nutrient metabolism inter alia through fermentation of carbohydrates. This results in the synthesis of butyrate, a short chain fatty acid (SCFA) which is an important energy source for colonocytes. It is known for its anti-inflammatory and anticancer properties. Intestinal microorganisms also have a positive influence on lipid metabolism. By means of the microbial proteinases and peptidases they enhance metabolism of proteins. Moreover, they are crucial for the synthesis of vitamin $\mathrm{K}, \mathrm{B}_{12}$, biotin and conjugated linoleic acids (CLA). They convert bile acids and are involved in the breakdown of various polyphenols [9, 10].

The capability of the gut microbiome to metabolize drugs has a profound impact on therapy for various diseases. For example, Eggerthella lenta present in the intestinal microbiota decreases the availability of digoxin by converting it to the reduced metabolite. Furthermore, microbiome may also induce the metabolism of drugs such as the anticancer drug known as irinotecan, which contributes to its toxicity causing diarrhea, inflammation, and anorexia [11]. The physical presence of the microbiota in the gastrointestinal (Gl) tract prevents pathogenic colonization by competing for attachment sites or nutrient sources, and by producing antimicrobial proteins (AMPs) such as cathelicidins, C-type lectins and defensins $[8,11]$. At the same time, the microbiota has an important immunomodulatory role. The components and the cell types from the immune system that participate in the immunomodulatory process include the gut-associated lymphoid tissues (GALT) [12].

In addition, the intestinal microbiota maintains the integrity of the gut barrier and structure of the Gl tract, due to substances produced by Bacteroides thetaiotaomicron, Lactobacillus rhamnosus GG and Akkermansia muciniphila. The gut barrier consists of epithelium, immune cells and intestinal bacteria. It performs a protective function against toxins and pathogens from the external environment. It is involved in the digestion, absorption of nutrients, fluids and electrolytes from food and fermentation of fiber to the compounds that intestinal bacteria feed on. Furthermore, the gut barrier secretes antibodies and participates in the transmission of signals to other cells and organs. The gut microbiota contributes to the structural development of the gut mucosa and can also modulate mucosal glycosylation patterns that are microbial attachment sites both at the cell surface and subcellular levels $[8,13,14]$.

The literature indicates that gut microbes have an extensive capacity to metabolize 430 environmental contaminants. Moreover, the bacteria-dependent metabolism of pollutants modulates the toxicity for the host. Conversely, environmental contaminants have been shown to alter the composition and the metabolic activity of gastrointestinal bacteria. The physiological consequences of these alterations have not been studied to date [15]. It is now well established that a healthy gut flora is largely responsible for the overall health of the host. There is a body of evidence that alterations of the microbiota composition (dysbiosis) and optimal functions caused by antibiotics, dietary ingredients, and lifestyle factors are associated with various metabolic and immune diseases and expansion of pathogenic populations [1-6, 16-23]. However, many studies have also shown a positive effect of some nutrients which have a profound impact on the development of a properly functioning microbiota.

\section{Antibiotic-related alteration}

Currently, there is substantial evidence that the composition of the gut microbiota may fluctuate in response to external factors such as antibiotics.

Panda et al. examined the fecal microbiota of $21 \mathrm{pa}-$ tients after 7-day treatment with commonly used antibiotic groups: fluoroquinolones and $\beta$-lactams. Both groups significantly decreased microbial diversity by $25 \%$ and reduced the core phylogenetic microbiota from 29 to 12 taxa. However, at the phylum level, these antibiotics increased the Bacteroidetes/Firmicutes ratio. At the species level, it was revealed that both antibiotic types increased the proportion of several unknown taxa belonging to the Bacteroides genus, a Gram-negative group of bacteria. Furthermore, the $\beta$-lactams increased the average microbial load two-fold. Eviction of microorganisms sensitive to these group of antibiotics provides space for resistant strains to overgrow and dominate the niche [24].

Another study focused on the long-term consequences of antibiotics on the composition, ecology, and resistance of the human gut microbiota. Several antibiotics are specifically active against anaerobic bacteria that dominate and play an important role in the human intestinal microbiota. One example is clindamycin, a relatively broad-spectrum antibiotic that primarily targets anaerobic bacteria. Clindamycin has demonstrated a large negative impact on the intestinal microbiota as seen by reduced resistance to colonization by patho- 
gens, leading to a high risk of pseudomembranous colitis due to Clostridium difficile overgrowth. Additional clindamycin-induced effects on the intestinal flora are gastritis and diarrhea. Disturbances of normal bowel function can lead to symptoms such as bloating and intestinal pain [25].

A research team from the University of Michigan examined 7 antibiotics from six classes on an animal model with the goal of differentially altering the microbiota and assessing their resistance to $C$. difficile colonization. They observed a high level of $C$. difficile colonization in mice treated with cefoperazone, metronidazole, and streptomycin. The greatest variation in the levels of C. difficile colonization was noted in mice treated with ampicillin. None of the mice that received ciprofloxacin were colonized. Only one of six mice receiving vancomycin was colonized, presumably due to residual vancomycin repressing $C$. difficile growth. Overall, these antibiotic perturbations provided different levels of colonization by C. difficile, which led to a hypothesis that the resulting communities varied in their composition [26].

Another investigation on a human model has produced results from the analysis of gut microbiota of four patients. Each patient presented their own initial microbiota and thus there was an individual response to antibiotic treatment with fluctuations in the bacterial diversity and composition for both total and active gut microbiota. These results highlight the importance of the initial microbial structure in shaping the changes in microbiota during the treatment. Within the patient group, three were treated with a bactericidal antibiotic, i.e. moxifloxacin, cefazolin and ampicillin/sulbactam, or amoxicillin, and one with the bacteriostatic combination of penicillin $\mathrm{G}$ and clindamycin. The patient treated with moxifloxacin had a decreased number of Faecalibacterium and Bacteroides genera. However, during the initial days of treatment, other butyrate-producing taxa such as Roseburia and Lachnospiraceae incertae sedis and the $\mathrm{H} 2$-consuming bacteria Blautia, Collinsella, and Bifidobacterium were present as active microbiota, obtaining energy sources for the colonocytes of the host. In the case of the patient treated with a combination of penicillin $G$ and clindamycin, there were marked decreases in Bacteroides and Blautia genera in the active microbiota. The patient treated with cefazolin and ampicillin/sulbactam showed a significant increase in Parabacteroides and Bacteroides genera. Also, the use of these antibiotics creates opportunistic infections by Escherichia spp. or Klebsiella spp. During the treatment of the fourth patient with amoxicillin, genera such as Escherichia or Klebsiella were almost eliminated, whereas Bacteroides, Blautia, and Faecalibacterium taxa proved less susceptible to treatment. Noteworthy is the fact that this bacterial profile, with Bacteroides, Blautia, and Faecalibacterium after the antibiotic stress, was found in the case of bactericidal agents but not when a bacteriostatic antimicrobial was used [27].

It has been established that a biochemical profile of antibiotic-related alteration has an impact on the gut flora as well. The antibiotic-treated microbiome liberates such mucosal carbohydrates as fucose and sialic acid. An increased level of these substances favors expansion of the opportunistic pathogens Salmonella typhimurium and $C$. difficile within the gut. Enterohaemorrhagic Escherichia coli has also been shown to access fucose or sialic acid liberated by the gut microbiota from mucins [28, 29].

To investigate the relationship of antibiotic exposure with microbiome perturbations in a murine model of growth promotion an animal model was used. Mice received sub-therapeutic antibiotic treatment (STAT), and were fed a high-fat diet (HFD). To characterize microbiota changes caused by STAT, the V4 region of the $16 \mathrm{~S}$ rRNA gene obtained from collected fecal samples was analyzed. By studying metabolic and hepatic functions and comparing them with the HFD-only control group of mice, it was found that this regimen promoted insulin resistance and hepatic steatosis. The research provides new information on the relationship between antibiotic therapy and metabolism as well as obesity pathogenesis [16].

In numerous studies, the interactions between complex intestinal microbial populations and the mammalian (rodents) immune system and how this relation is perturbed by the antibiotic administration have been defined [7, 8, 15, 17, 24, 27]. Amoxicillin treatment essentially eradicates the small intestine and reduces the density of colonic anaerobic and aerobic bacteria. Amoxicillin-induced microbiotal changes reduce expression of MHC class I and II genes in Lactobacillus spp. in the small and large intestine, reduce AMP expression in the proximal intestine, and increase mast cell protease expression in the distal small intestine. Treatment of mice with metronidazole, neomycin, and vancomycin diminishes the expression of other important immune factors. This enhances vancomycin-resistant Enterococcus (VRE) colonization of the intestinal tract. Changes in the microbiota following antibiotic treatment also affect immune responses to viral infection. Neomycin diminishes the expression of the two pro-cytokines, impairing responses against influenza viruses. Another study has shown that antibiotic treatment enhances immune responses against the mouse mammary tumor virus (MMTV). Some bacteria have the ability to modulate inflammatory processes. Antibiotic administration can alter intestinal commensal bacterial populations and, as a consequence, modify immune defenses, leading, 
in some cases, to detrimental effects on health. Viral and bacterial infections or allergies can be promoted by antibiotic-induced changes in the microbiota [17].

As described above, antibiotics can have a negative impact on the microbiota, immunity and health. Avoidance of antibiotics, however, is often not feasible, because many infections can only be overpowered with antibiotic treatment. For this reason, new strategies have to be tested to counteract the negative effect of antibiotics on the microbiota and immune responses. To reduce unfavorable alterations in human gut microbiota caused by antibiotic therapy, currently commercial probiotics have been used widely. Mostly such products contain such strains as Lactobacillus spp., Bifidobacterium spp. as well as prebiotics such as fructooligosaccharides. It is also recommended to increase the intake of traditional fermented vegetables and dairy products. Fecal microbiota transplantation is becoming increasingly accepted as an effective and safe intervention in patients with relapsing Clostridium difficile infection, inflammatory bowel disease, obesity and metabolic syndrome, likely due to the restoration of a disrupted microbiome [18, 19].

\section{The influence of dietary ingredients}

The microbiome in humans differs among cultures and geographic regions. Also, it is not constant during the lifespan, but rather changes with age. Diet is a particularly important factor in determining the microbiota composition of the gut. Thus, vegans, vegetarians, and omnivores have distinct microbiomes. Total counts of Bacteroides spp., Bifidobacterium spp., Escherichia coli and Enterobacteriaceae spp. were significantly lower in vegan samples than in controls. In contrast, total E. coli biovars, Klebsiella spp., Enterobacter spp., other Enterobacteriaceae, Enterococcus spp., Lactobacillus spp., Citrobacter spp. and Clostridium spp. were similar. The total microbial count did not differ between the groups. In addition, subjects on a vegan or vegetarian diet showed significantly lower stool $\mathrm{pH}$ than did controls, and stool pH and counts of E. coli and Enterobacteriaceae were significantly correlated across all subgroups. Maintaining a strict vegan or vegetarian diet results in a significant shift in the microbiota while total cell numbers remain unaltered. An animal-based diet increased the abundance of bile-tolerant microorganisms, including Alistipes, Bilophila, and Bacteroides, whereas it decreased the levels of Firmicutes that metabolize dietary plant polysaccharides [1, 20]. Moreover, the intestinal microbiota of omnivores produces significantly more trimethylamine- $\mathrm{N}$-oxide (TMAO) from choline and L-carnitine present in eggs and red meat than intestinal microbiota of vegans or vegetarians through a mi- crobiota-dependent mechanism. An increased level of TMAO promotes the risk of atherosclerosis. Therefore, intestinal microbiota may thus participate in the wellestablished link between increased red meat or egg consumption and cardiovascular disease occurrence [21].

It has been demonstrated that even a short-term change of diet can alter the microbial community structure. A gluten-free diet (GFD) is the most commonly adopted special diet worldwide. It was concluded that 13 weeks of a GFD had a significant effect on the microbiome diversity between the control and the examined group of patients, and also large differences in pathway activities were observed. Moreover, there was an increased number of $E$. coli and Enterobacteriaceae including opportunistic pathogens [22].

The western diet (high in animal fat and protein and low in fiber) leads to a decrease in total bacteria and beneficial Bifidobacterium and Eubacterium, together with increased production of cancer-promoting nitrosamines and inflammation. The Mediterranean diet (high in fiber and antioxidants and low in red meat) is related to increased levels of fecal short-chain fatty acids (SCFAs), Bifidobacteria, Lactobacilli, Eubacteria, Bacteroides and Prevotella [23].

A primary means by which the intestine is protected from its microbiota is via multilayered mucus structures that cover the intestinal surface, thus allowing the vast majority of gut bacteria to be kept at a safe distance from the epithelial cells that line the intestine. Thus, detergent-like emulsifiers that disrupt mucus-bacterial interactions have the potential to promote diseases associated with gut inflammation. Chassaing and colleagues, using an animal model, demonstrated that the emulsifiers carboxymethylcellulose and polysorbate-80, commonly used in processed food, altered fecal levels of short-chain fatty acids, including decreased levels of butyrate. They also altered the bile acid level, which influences microbiota composition. The same study suggested that emulsifier-induced changes in microbiota play a role in driving the inflammation and metabolic changes promoted by these food additives [30].

It has also been demonstrated that artificial sweeteners such as saccharin, sucralose, aspartame, and acesulfame $\mathrm{K}$ alter gut microbial communities, leading to glucose intolerance in both mice and humans. To test whether changes in microbial composition, induced by the consumption of non-caloric artificial sweeteners (NAS), led to glucose intolerance, intestinal microbiota from NAS-fed or control mice was transferred into germ-free mice, which were maintained under aseptic conditions, making their gastrointestinal tracts completely sterile. The results indicated that NAS consumption directly altered microbial composition and me- 
tabolism, leading to important downstream metabolic effects. The same group of scientists found a correlation between NAS consumption and gut microbiome alteration in humans. In their study, 172 randomly selected individuals from a cohort showed intestinal microbial changes characterized by increases in Enterobacteriaceae, Deltaproteobacteria, and Actinobacteria phyla. In terms of the effect on the animal microbiome, it was found that the total number of anaerobes in the gut microbiota of rats decreased significantly in those fed with sucralose plus maltodextrin. At the lowest dose, total anaerobes were reduced by approximately $50 \%$ compared with controls. Bifidobacterium, Lactobacillus, and Bacteroides were significantly reduced at the lowest dose as well. Simultaneously, increased secretion of incretin was observed when sucralose was combined with glucose. As a result, decreased mobility of the gastrointestinal tract was observed [31].

Recently, it has been shown that two clinically effective drugs for treating diabetes - metformin and berberine - exert their actions through modulating the gut microbiota and may contribute to beneficial effects on the host. It was demonstrated that they similarly shifted the overall structure of the gut microbiota in rats. Both drugs showed reverting effects on the highfat diet-induced structural changes of gut microbiota. The diversity of the gut microbiota was significantly reduced by both berberine and metformin treatments. Sixty out of the 134 operational taxonomic units were decreased by both drugs. Conversely, short-chain fatty acid (SCFA)-producing bacteria - Allobaculum, Bacteroides, Blautia, Butyricicoccus, and Phascolarctobacterium - were markedly increased. Although both berberine and metformin increased the SCFA-producing bacteria and reduced the gut microbial diversity, metformin did so to a lesser extent. The selective enrichment of the SCFA-producing bacteria and reduction of gut microbial diversity by both drugs may be shared mechanisms for improving the gastrointestinal health, and may mediate their beneficial effects on the host, particularly in metabolic and cardiovascular diseases [32].

Curcumin is the most active constituent of the ground rhizome of the plant Curcuma longa, which has been demonstrated to have anti-inflammatory, anti-oxidative and anti-proliferative properties. It has been found that curcumin has a substantial influence on the gut microbiota. Curcumin increased bacterial richness in mice. Furthermore, it prevented the age-related decrease in alpha diversity, increased the relative abundance of the order Lactobacillales, and decreased that of the order Coriobacteriales. The maintenance of a more diverse colonic microbial ecology correlates with a beneficial effect of curcumin on tumorigenesis [33].
Data indicate a link between omega-3 circulating intake and microbiome composition that suggests the potential use of omega-3 supplementation to improve the microbiome composition. Walnuts are rich in omega-3 fatty acids, phytochemicals, and antioxidants, making them unique compared to other foods. Consumption of walnuts has been associated with health benefits including a reduced risk of heart disease and cancer. One potential mechanism by which walnuts may exert their health benefit is through modifying the gut microbiome. The study of Byerleya et al. identified the changes in the gut microbial communities that occur following the inclusion of walnuts in the diet of rats. Intestinal samples were collected from the descending colon, the DNA was isolated, and the V3-V4 hypervariable region of the 16S rRNA gene was deep sequenced on an Illumina MiSeq for characterization of the gut microbiota. Animals consuming walnuts displayed significantly greater species diversity. Walnuts increased the abundance of Firmicutes and reduced the abundance of Bacteroidetes. It also enriched the microbiota for probiotic-type bacteria including Lactobacillus, Ruminococcaceae, and Roseburia while significantly reducing Bacteroides and Anaerotruncus [34, 35].

Notably, the microbiome of a person can be altered by microbial metabolites from non-digestible food ingredients. In particular 'non-digestible' dietary carbohydrates including polysaccharides, oligosaccharides, lignin and associated plant material are the preferred energy source for colonic microbiota. Nowadays, researchers are exploring the effects of microbial metabolites from polyphenols on the health of the mucosal epithelial cells, intestine and immune system. Polyphenols are known for their antioxidant capacity as well as anti-inflammatory and anti-proliferative activity. In the colon, they undergo extensive bioconversion and metabolism by the gut microbiota. They are deconjugated by bacterial glycosidases, glucuronidases, and sulfatases and further fermented to a wide range of low-molecular-weight phenolic acids. Thus, the gut microbiota plays a key role in the bioavailability of polyphenols and has been shown to modulate the health-promoting activity through the transformation to more active derivatives. Compounds derived from microbial metabolism of proanthocyanidins and polyphenols have anti-inflammatory properties. These microbial metabolites could be among the new generation of therapeutic agents for the management of immune-inflammatory diseases as well as for dampening the inflammatory response to bacterial antigens, which may have implications for chronic inflammatory or autoimmune diseases such as inflammatory bowel disease. 
However, although the great range of health-promoting activities of dietary polyphenols has been widely investigated, their effect on the modulation of the gut ecology and the two-way relationship polyphenols microbiota is still poorly understood. There is evidence from in vitro animal and human studies that certain doses of selected polyphenols may modify the gut microbial composition, and while certain bacterial groups can be inhibited, others can thrive in the available niche of the ecosystem. Phenolic compounds alter the gut microbiota and, consequently, alter the Bacteroides/ Firmicutes balance. For example, flavan-3-ol monomers such as (-)epicatechin and (+)catechin may be capable of influencing the large intestinal bacterial population even in the presence of other nutrients, such as carbo- hydrates and proteins. It was found that (+)catechin significantly inhibited the growth of Clostridium histolyticum and enhanced the growth of E. coli and members of the Clostridium coccoides-Eubacterium rectale group, while the growth of Bifidobacterium and Lactobacillus spp. remained relatively unaffected. It was also documented that a proanthocyanidin-rich extract from grape seeds given to healthy adults for 2 weeks was able to significantly increase the number of Bifidobacteria. A human intervention study indicated that consumption of red wine polyphenols significantly increased the number of Enterococcus, Prevotella, Bacteroides, Bifidobacterium, Bacteroides uniformis, Eggerthella lenta and Blautia coccoides-E. rectale group while the quantity of Lactobacillus spp. was unaltered [36, 37].

Table I. Summary of the effects of antibiotics and dietary compounds on gut microbiota

\begin{tabular}{|c|c|c|}
\hline Factor & Alteration & Clinical relevance \\
\hline $\begin{array}{l}\text { Fluoroquinolones and } \\
\beta \text {-lactams [24] }\end{array}$ & $\begin{array}{l}\text { Decreased microbial diversity, reduced number } \\
\text { of taxa, changed Bacteroidetes/Firmicutes ratio, } \\
\text { increased average microbial load [24] }\end{array}$ & $\begin{array}{c}\text { Systematic use of antibiotics can reshape the } \\
\text { microbiota in favor of resistant bacterial strains in } \\
\text { the long term [24] }\end{array}$ \\
\hline Clindamycin [25] & $\begin{array}{l}\text { Reduced resistance to colonization } \\
\text { by pathogens [25] }\end{array}$ & $\begin{array}{l}\text { High risk of pseudomembranous colitis due to } \\
\text { Clostridium difficile overgrowth, gastritis and } \\
\text { diarrhea, bloating and intestinal pain [25] }\end{array}$ \\
\hline $\begin{array}{l}\text { Moxifloxacin, cefazolin, } \\
\text { ampicillin/sulbactam, } \\
\text { amoxicillin, penicillin G/ } \\
\text { clindamycin [27] }\end{array}$ & Qualitative and quantitative changes in genera [27] & $\begin{array}{l}\text { Increased risk of opportunistic infections caused by } \\
\text { Escherichia spp. or Klebsiella spp. [27] }\end{array}$ \\
\hline $\begin{array}{l}\text { Antibiotic treatment } \\
{[17,28,29]}\end{array}$ & $\begin{array}{c}\text { Fucose, sialic acid liberated from mucins }[28,29] \text {. } \\
\text { Alteration of intestinal commensal bacteria causes } \\
\text { immune defense modification }[17]\end{array}$ & $\begin{array}{l}\text { Expansion of the opportunistic pathogens } \\
\text { Salmonella typhimurium, C. difficile and E. coli [28, } \\
\text { 29]. Allergy and viral infection promotion [17] }\end{array}$ \\
\hline $\begin{array}{l}\text { Choline (eggs), L-carnitine } \\
\text { (red meat) [21] }\end{array}$ & Increased level of TMAO [21] & Increased risk of atherosclerosis [21] \\
\hline Western diet [23] & $\begin{array}{l}\text { Decrease in total bacteria and beneficial } \\
\text { Bifidobacterium and Eubacterium [23] }\end{array}$ & $\begin{array}{c}\text { Increased production of cancer-promoting } \\
\text { nitrosamines and inflammation [23] }\end{array}$ \\
\hline $\begin{array}{l}\text { Emulsifiers: } \\
\text { carboxymethylcellulose and } \\
\text { polysorbate- } 80[30]\end{array}$ & $\begin{array}{l}\text { Decreased levels of butyrate, altered bile } \\
\text { acid level [30] }\end{array}$ & $\begin{array}{c}\text { Increased risk of inflammation and metabolic } \\
\text { changes [30] }\end{array}$ \\
\hline Artificial sweeteners [31] & $\begin{array}{c}\text { Increase in Enterobacteriaceae, Deltaproteobacteria, } \\
\text { and Actinobacteria phylum. Decrease in } \\
\text { Bifidobacterium, Lactobacillus, and Bacteroides, } \\
\text { increased incretin secretion [31] }\end{array}$ & $\begin{array}{l}\text { Glucose intolerance, decreased motility and } \\
\text { visceral hypersensitivity [31] }\end{array}$ \\
\hline $\begin{array}{l}\text { Metformin and berberine } \\
\text { [32] }\end{array}$ & $\begin{array}{c}\text { Structural changes of gut microbiota, } \\
\text { significantly reduced diversity of gut microbiota, } \\
\text { increase in short-chain fatty acid (SCFA)-producing } \\
\text { bacteria [32] }\end{array}$ & $\begin{array}{l}\text { Metabolic disease prevention, } \\
\text { anti-obesity effect [32] }\end{array}$ \\
\hline Curcumin [33] & $\begin{array}{l}\text { Increased bacterial richness, increased } \\
\text { Lactobacillales, and decreased Coriobacteriales [33] }\end{array}$ & Beneficial effect on tumorigenesis [33] \\
\hline $\begin{array}{l}\text { Walnuts, omega-3 fatty } \\
\text { acids }[34,35]\end{array}$ & $\begin{array}{l}\text { Greater species diversity, enriched the microbiota } \\
\text { for probiotic-type bacteria }[34,35]\end{array}$ & Reduced risk of heart disease and cancer [34, 35] \\
\hline $\begin{array}{l}\text { Polyphenols and } \\
\text { proanthocyanidins }[36,37]\end{array}$ & $\begin{array}{c}\text { Stimulation of growth of beneficial bacteria } \\
\text { and inhibition of pathogenic bacteria, exerting } \\
\text { prebiotic-like effects, beneficial compounds from } \\
\text { their microbial metabolism }[36,37]\end{array}$ & $\begin{array}{l}\text { Management of immune-inflammatory diseases, } \\
\text { chronic inflammatory, autoimmune diseases such } \\
\text { as inflammatory bowel disease }[36,37]\end{array}$ \\
\hline
\end{tabular}




\section{Summary}

The microbes that reside in the human gastrointestinal tract comprise a dynamic community that changes throughout the lifespan of an individual and due to the diet and treatment agents. A short summary of the positive and negative effect of antibiotics and dietary compounds on the microbiota and its clinical relevance is presented below in the Table I.

Therefore, there is a body of evidence suggesting that the gut microbiota is a major, still underestimated element that must be considered as an important factor shaping human health.

\section{Conflict of interest}

The authors declare no conflict of interest.

\section{References}

1. Thomas S, Izard J, Walsh E, et al. The host microbiome regulates and maintains human health: a primer and perspective for non-microbiologists. Cancer Res 2017; 77: 1783-812.

2. Matijaši $M$, Meštrović T, Perić $M$, et al. Modulating composition and metabolic activity of the gut microbiota in IBD patients. Int J Mol Sci 2016; 17: 578.

3. Distrutti E, Monaldi L, Ricci P, et al. 2016 irritable bowel syndrome: global view gut microbiota role in irritable bowel syndrome: new therapeutic strategies. World J Gastroenterol 2016; 22: 2219-41.

4. Forsythe P, Kunze W. Voices from within: gut microbes and the CNS. Cell Mol Life Sci 2013; 70: 55-69.

5. Burke DG, Fouhy F, Harrison M, et al. The altered gut microbiota in adults with cystic fibrosis. BMC Microbiol 2017; 17: 58.

6. Kaufman D, Kelly J, Curhan G, et al. Oxalobacter formigenes may reduce the risk of calcium oxalate kidney stones. J Am Soc Nephrol 2008; 19: 1197-203.

7. Sela DA, Mills D. Nursing our microbiota: molecular linkages between bifidobacteria and milk oligosaccharides. Trends Microbiol 2011; 18: 298-307.

8. Jandhyala S, Talukdar R, Subramanyam C, et al. Role of the normal gut microbiota. World J Gastroenterol 2015; 21: 8787-803.

9. Ozdal T, Sela DA, Xiao J, et al. The reciprocal interactions between polyphenols and gut microbiota and effects on bioaccessibility. Nutrients 2016; 8: 78.

10. Klaassen CD, Yue Cui JY. Review: mechanisms of how the intestinal microbiota alters the effects of drugs and bile acids. Drug Metab Dispos 2015; 43: 1505-21.

11. Spanogiannopoulos P, Bess EN, Carmody RN, et al. The microbial pharmacists within us: a metagenomic view of xenobiotic metabolism. Nat Rev Microbiol 2016; 14: 273-87.

12. Thursby E, Juge N. Introduction to the human gut microbiota. Biochem J 2017; 474: 1823-36.

13. Bron PA, Kleerebezem M, Brummer RJ, et al. Can probiotics modulate human disease by impacting intestinal barrier function? Br J Nutr 2017; 117: 93-107.

14. Marlicz W, Yung DE, Skonieczna-Żydecka K, et al. From clinical uncertainties to precision medicine: the emerging role of the gut barier and microbiome in small bowel functional disease. Expert Rev Gastroenterol Hepatol 2017; 11: 961-78.

15. Claus SP, Guillou H, Ellero-Simatos S. The gut microbiota: a major player in the toxicity of environmental pollutants? NPJ Biofilms Microbiomes 2017; 3: 17001.

16. Mahana D, Trent C, Zachary D. Kurtz Z, et al. Antibiotic perturbation of the murine gut microbiome enhances the adiposity, insulin resistance, and liver disease associated with high-fat diet. Genome Med 2016; 8: 48.

17. Ubeda C, Pamer EG. Antibiotics, microbiota, and immune defense. Trends Immunol 2012; 33: 459-66.

18. Gupta S, Allen-vercoe E, Petrof EO. Fecal microbiota transplantation: in perspective. Ther Adv Gastroenterol 2016; 9: 229-39.

19. Leszczyszyn JJ, Radomski M, Leszczyszyn AM. Intestinal microbiota transplant - current state of knowledge. Reumatologia 2016; 54: 24-8.

20. Zimmer J, Lange B, Frick JS, et al. A vegan or vegetarian diet substantially alters the human colonic faecal microbiota. Eur J Clin Nutr 2011; 66: 53-6.

21. Koeth RA, Wang Z, Levison BS, et al. Intestinal microbiota metabolism of L-carnitine, a nutrient in red meat, promotes atherosclerosis. Nat Med 2013; 19: 576-85.

22. Bonder MJ, Tigchelaar EF, Cai X, et al. The influence of a shortterm gluten-free diet on the human gut microbiome. Genome Med 2016; 8: 45.

23. Del Chierico F, Vernocchi P, Dallapiccola B, et al. Mediterranean diet and health: food effects on gut microbiota and disease control. Int J Mol Sci 2014; 15: 11678-99.

24. Panda S, El Khader I, Casellas F, et al. Short-term effect of antibiotics on human gut microbiota. PLoS One 2014; 9: e95476.

25. Jernberg C, Löfmark S, Edlund C, et al. Long-term impacts of antibiotic exposure on the human intestinal microbiota. Microbiology 2010; 156: 3216-23.

26. Schubert A, Sinani H, Schloss. PD. Antibiotic-induced alterations of the murine gut microbiota and subsequent effects on colonization resistance against Clostridium difficile. MBio 2015; 6: e00974-15.

27. Perez-Cobas AE, Artacho A, Knecht $\mathrm{H}$, et al. Differential effects of antibiotic therapy on the structure and function of human gut microbiota. PLoS One 2013; 8: e80201.

28. Ng K, Ferreyra J, Higginbottom S, et al. Microbiota-liberated host sugars facilitate post-antibiotic expansion of enteric pathogens. Nature 2013; 502: 96-99.

29. Huang YL, Chassard C, Hausmann M, et al. Sialic acid catabolism drives intestinal inflammation and microbial dysbiosis in mice. Nat Commun 2015; 6: 8141.

30. Chassaing B, Koren O, Goodrich JK, et al. Dietary emulsifiers impact the mouse gut microbiota promoting colitis and metabolic syndrome. Nature 2016; 519: 404-13.

31. Spencer M, Gupta A, Van Dam L, et al. Artificial sweeteners: a systematic review and primer for gastroenterologists. J Neurogastroenterol Motil 2016; 22: 168-80.

32. Zhang $X$, Zhao $Y, X u$ J, et al. Modulation of gut microbiota by berberine and metformin during the treatment of high-fat dietinduced obesity in rats. Sci Rep 2015; 5: 14405.

33. McFadden R, Larmonier C, Shehab K, et al. The role of curcumin in modulating colonic microbiota during colitis and colon cancer prevention. Inflamm Bowel Dis 2015; 21: 2483-94. 
34. Byerleya LO, Samuelson D, Blanchard E, et al. Changes in the gut microbial communities following addition of walnuts to the diet. J Nutr Biochem 2017; 48: 94-102.

35. Sardecka I, Krogulska A, Toporowska-Kowalska E. The influence of dietary immunomodulatory factors on development of food allergy in children. Postep Dermatol Alergol 2017; 34: 89-96.

36. Jacobs DM, Gaudier E, van Duynhoven J, et al. Non-digestible food ingredients, colonic microbiota and the impact on gut health and immunity: a role for metabolomics. Curr Drug Metab 2009; 10: 41-54.

37. Cardona F, Andrés-Lacuevac C, Tulipani S, et al. Benefits of polyphenols on gut microbiota and implications in human health. J Nutr Biochem 2013; 24: 1415-22.

Received: 20.11.2017

Accepted: 24.04 .2018 